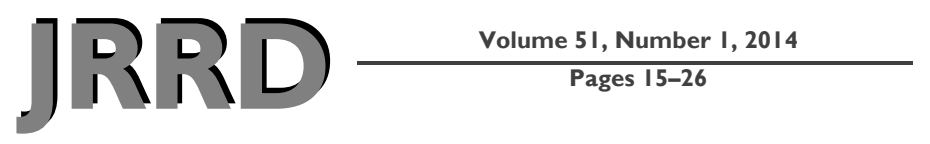

\title{
User ratings of prosthetic usability and satisfaction in VA study to optimize DEKA Arm
}

\author{
Linda Resnik, PT, PhD; ${ }^{1-2 *}$ Matthew Borgia, AM ${ }^{\mathbf{1}}$ \\ ${ }^{1}$ Providence Department of Veterans Affairs Medical Center, Providence, RI; ${ }^{2}$ Department of Health Services, Policy, \\ and Practice, Brown University, Providence, RI
}

\begin{abstract}
The Department of Veterans Affairs study to optimize the DEKA Arm provided feedback to inform optimization of the gen 2 (second-generation) prototype and evaluate the gen 3 (third-generation) prototype. This article summarizes recommendations to improve gen 2 and reports satisfaction and usability ratings of gen 2 and gen 3. Data were collected from 39 subjects; 37 subjects were included in this analysis. Of the subjects, 24 were fit with gen 2 (8 radial configuration [RC], 6 humeral configuration [HC], and 10 shoulder configuration [SC]), 13 were fit with gen 3 ( 4 RC, 5 HC, and 4 SC), and 5 were fit with both. Usability and satisfaction were evaluated using the Trinity Amputation and Prosthesis Experience Scale (TAPES) and study-specific usability and satisfaction scales. Descriptive statistics were examined and prototypes compared using Wilcoxon rank-sum. Results were stratified by configuration level and outcomes compared by prototype. Satisfaction and usability were greater for gen 3 than gen 2. Overall TAPES scores were similar; however, scores of the TAPES aesthetic satisfaction subscale were higher for gen 3. Compared with gen 2 users, gen 3 users were more satisfied with appearance, grips, and doffing and rated overall usability higher. Features of gen 3, including weight, external cables and wires, hand covering, and fingernails, would benefit from further optimization.
\end{abstract}

Key words: amputation, assistive technology, DEKA Arm, optimization, outcome assessment, prosthesis, satisfaction, upper limb, usability, Veterans.

\section{INTRODUCTION}

Commercially available upper-limb prostheses can be controlled through one of two means. They can be body- powered, using a harnessing cable operated through shoulder motion, or they can be controlled by myoelectric recordings from the residual muscles. Hybrid devices use a combination of body-powered and myoelectric controls. These controls can be used to operate one prosthetic movement at a time. Currently available prostheses are also limited in the types of motions that they can perform. None include powered wrist flexion or extension, powered humeral rotation, or powered shoulder movement. Furthermore, there are no devices on the market that allow multiple simultaneous joint movements.

A recent Inspector General report found that only 70 percent of new combat Veterans were satisfied with their current upper-limb prosthesis [1]. This report builds upon ample evidence that suggests that people with

Abbreviations: CFI $=$ Center for the Intrepid, DARPA $=$ Defense Advanced Research Projects Agency, EMG = myoelectrode, gen 2 = second-generation DEKA Arm, gen 3 = third-generation DEKA Arm, HC = humeral configuration, IMU = inertial measurement unit, LUI $=$ Luke User Interface, NYHHS = New York Harbor Healthcare System, RC = radial configuration, RR\&D = Rehabilitation Research and Development, SC = shoulder configuration, $\mathrm{SD}=$ shoulder disarticulation, $\mathrm{TAPES}=$ Trinity Amputation and Prosthesis Experience Scale, $\mathrm{TH}=$ transhumeral, $\mathrm{TR}=$ transradial, VA = Department of Veterans Affairs.

*Address all correspondence to Linda Resnik, PT, PhD; Providence VA Medical Center, 830 Chalkstone Ave, Providence, RI 02908; 401-273-7100, ext 2368.

Email: Linda.Resnik@va.gov

http://dx.doi.org/10.1682/JRRD.2013.02.0056 
upper-limb amputation are not satisfied with available technology. As a result, many reject using a prosthesis altogether [2-3]. Rates of abandonment are higher for those with more proximal levels of limb loss, with persons with transradial (TR) amputation having the lowest rates (6\%), and persons with transhumeral (TH) amputation and shoulder disarticulation (SD) reporting rates of 57 and 60 percent, respectively [2-3]. There are many reasons for dissatisfaction with currently available devices [4-9]. Development of new and better upperlimb prosthetic technology was identified as a high priority by the participants at the 2006 State-of-the-Science Meeting in Prosthetics and Orthotics [10]. Improving care for people with upper-limb amputation has been a strong priority for the Department of Defense and the Department of Veterans Affairs (VA) for the past decade.

The development of the prototype of the DEKA prosthetic arm system was funded by the Defense Advanced Research Projects Agency's (DARPA's) Revolutionizing Prosthetics program in 2006 [11] with a goal of drastically improving the state of the art in upper-limb prosthetics. Two years later, DEKA had built and tested the first-generation DEKA Arm (gen 1) and had developed the initial gen 2 (second-generation) prototype. The gen 2 DEKA Arm was designed as an experimental platform, and as such, it included many test features that had not yet been finalized or miniaturized. In 2008, the VA and DARPA entered into a Memorandum of Agreement to collaborate on a study to optimize the DEKA Arm. Subsequently, VA Rehabilitation Research and Development (RR\&D) funded the multisite VA study to optimize the gen 2 DEKA Arm (Optimization Study). VA subject and clinician feedback about gen 2 was analyzed on an ongoing basis and shared with DEKA. This feedback was used in refining the gen 2 prototype and finalizing the gen 3 (third-generation) prototype, which had major hardware, software, and design changes. VA subject and clinician feedback about the gen 3 DEKA Arm was gathered in the next phase of the Optimization Study, which began in 2011.

The gen 2 and gen 3 DEKA Arm prototypes are described in detail elsewhere [12]. Briefly, the DEKA Arm (both gen 2 and gen 3) is available in three configurations: the radial configuration (RC), for people with TR amputation; the humeral configuration (HC), for people with $\mathrm{TH}$ amputation; and the shoulder configuration (SC), for people with very short TH amputation or ampu- tation at the SD and scapulothoracic level. Figure 1 shows gen 2 and gen 3 SC prototypes.

The SC DEKA Arm has 10 powered degrees of freedom (20 movements) and additional passive degrees of freedom [13-14]. A major change in the gen 3 was the inclusion of a compound wrist that combined the movements of radial deviation with wrist flexion and the movements of ulnar deviation with wrist extension. Both prototypes supported the use of up to six preprogrammed grip patterns: power grip, tool grip, chuck grip, lateral pinch, fine pinch open, and fine pinch closed. Users selected the desired grip directly or by toggling through the grips. Three grips were modified in gen 3 by adding a new feature, called a detent, that allowed users to separate the positioning and/or stabilizing and grasping aspects of grip from the precision portion.

All levels of the DEKA Arm used control inputs for the hand and wrist. At the HC and SC levels, the control scheme had dual modes enabling the user to switch between a "hand mode" of operation (to control movements of the hand and wrist) and an "arm mode" of operation. In gen 3, changes were made to enable up to three movements of the hand and/or wrist to also be available in arm mode if sufficient control inputs were available.

The SC DEKA Arm employed end-point control to enable simultaneous, coordinated movement of the prosthesis to bring the terminal device (the end point) to a desired position in space. During the Optimization Study, there were three different versions of end-point control, each with some differences in movement trajectories and features.

Users controlled prosthetic movements with a combination of foot controls, myoelectrodes (EMGs), pneumatic bladders, or manual switches. Three iterations of foot controls (Figure 2) were used: force sensitive resistors; inertial measurement units (IMUs) during gen 2 (IMU-1); and a refined version of the IMU in gen 3 (IMU-2) that had new features, including the ability to detect walking motion and automatically put the arm into standby (called walk detect).

The DEKA Arm was battery-powered by a rechargeable battery typically worn on a holster on a belt around the waist or on the back. The gen 3 for HC and SC had the potential to include an internal battery. The DEKA Arm made a variety of beeping sounds to notify users of powering on and off, low battery, and system faults. In addition, an audible tone and a vibratory tactor "buzzed" to indicate changes between hand and arm mode, moving 

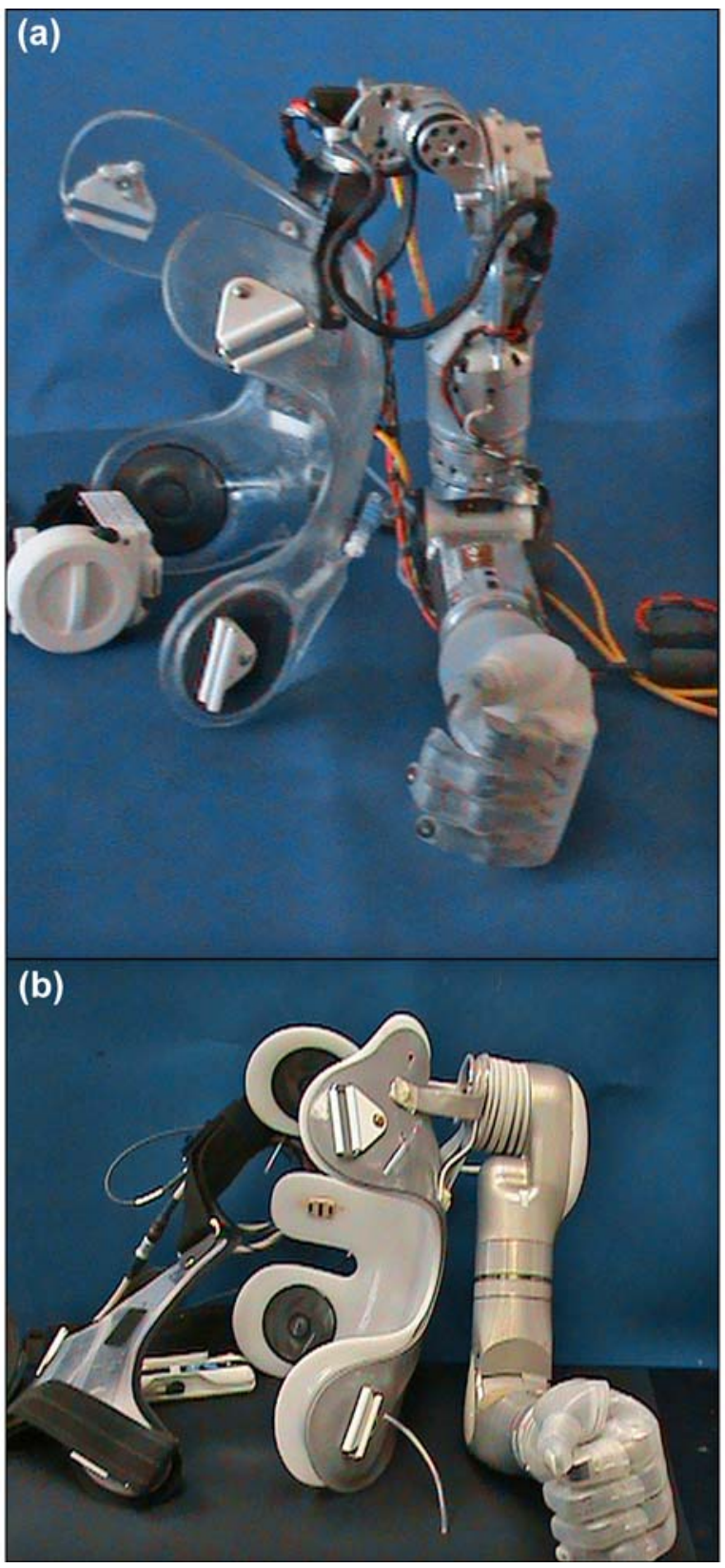

Figure 1.

(a) Second-generation DEKA Arm shoulder configuration (SC) on socket. (b) Third-generation DEKA Arm SC on socket.

into or out of standby, changing grip, and changing grip pressure. The Luke User Interface (LUI) introduced during the gen 2 studies displayed information to the user about grip, mode, power, battery charge, and system faults (Figure 3). The gen 3 replaced the LUI with a wrist display embedded on the dorsal wrist (Figure 3) that had light-emitting diode displays for grip, low battery, mode

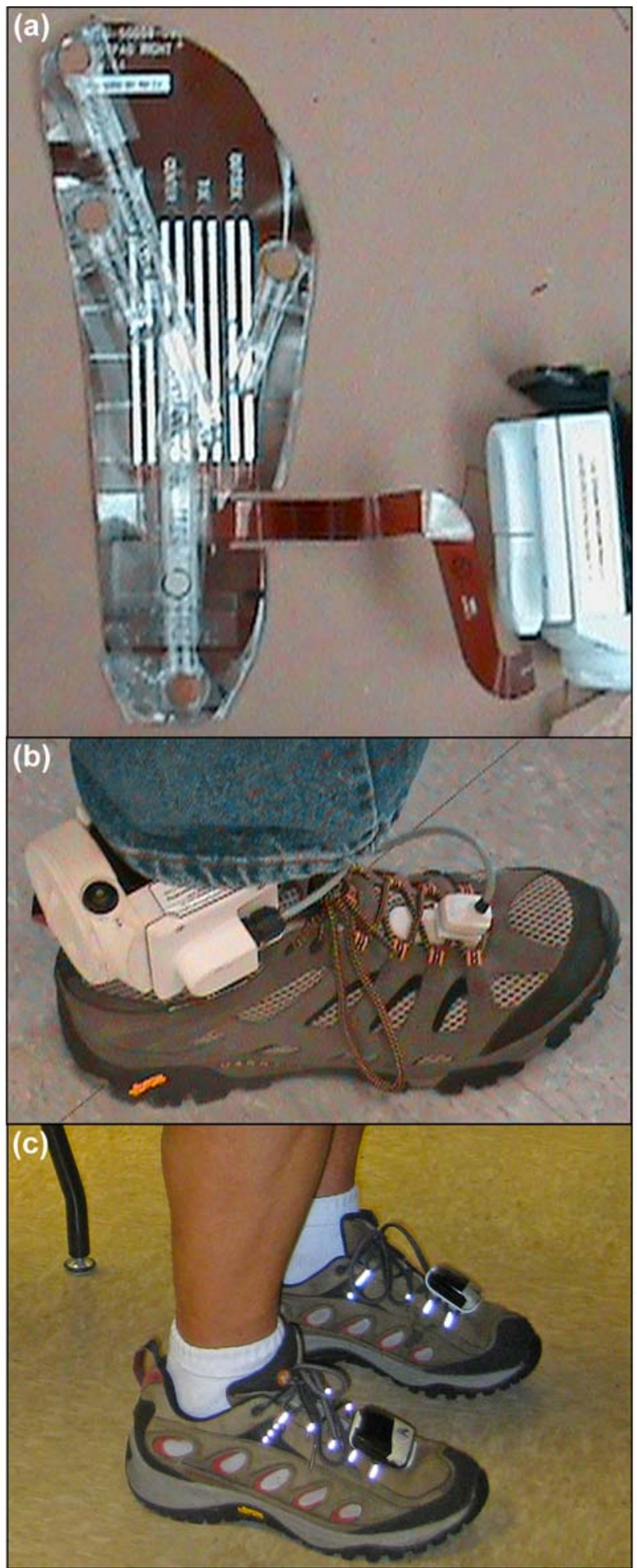

Figure 2.

Three iterations of foot controls. (a) Force sensitive resistor footpad wired to arm control interface (ACl). (b) Inertial measurement unit (IMU)-1 and ACI unit. (c) IMU-2 worn on shoelaces. 
notification, and system faults, as well as indicators for walk detect and detection of over-angle limits for the IMU (that temporarily disabled the IMU-2).

DEKA designed inflatable socket bladders or actuators to be embedded inside TH sockets using a special design aimed at increasing skeletal stabilization [15] or to be used inside $\mathrm{X}$-frame sockets to provide pressure relief and greater stability. During the gen 3 phase, DEKA introduced a dynamic socket controller that could regulate inflation of the socket bladders through independent pneumatic channels using the touch of one or more buttons.

The VA study collected both qualitative and quantitative data on subject and clinician attitudes and opinions about the usability of the DEKA Arm. This article summa-
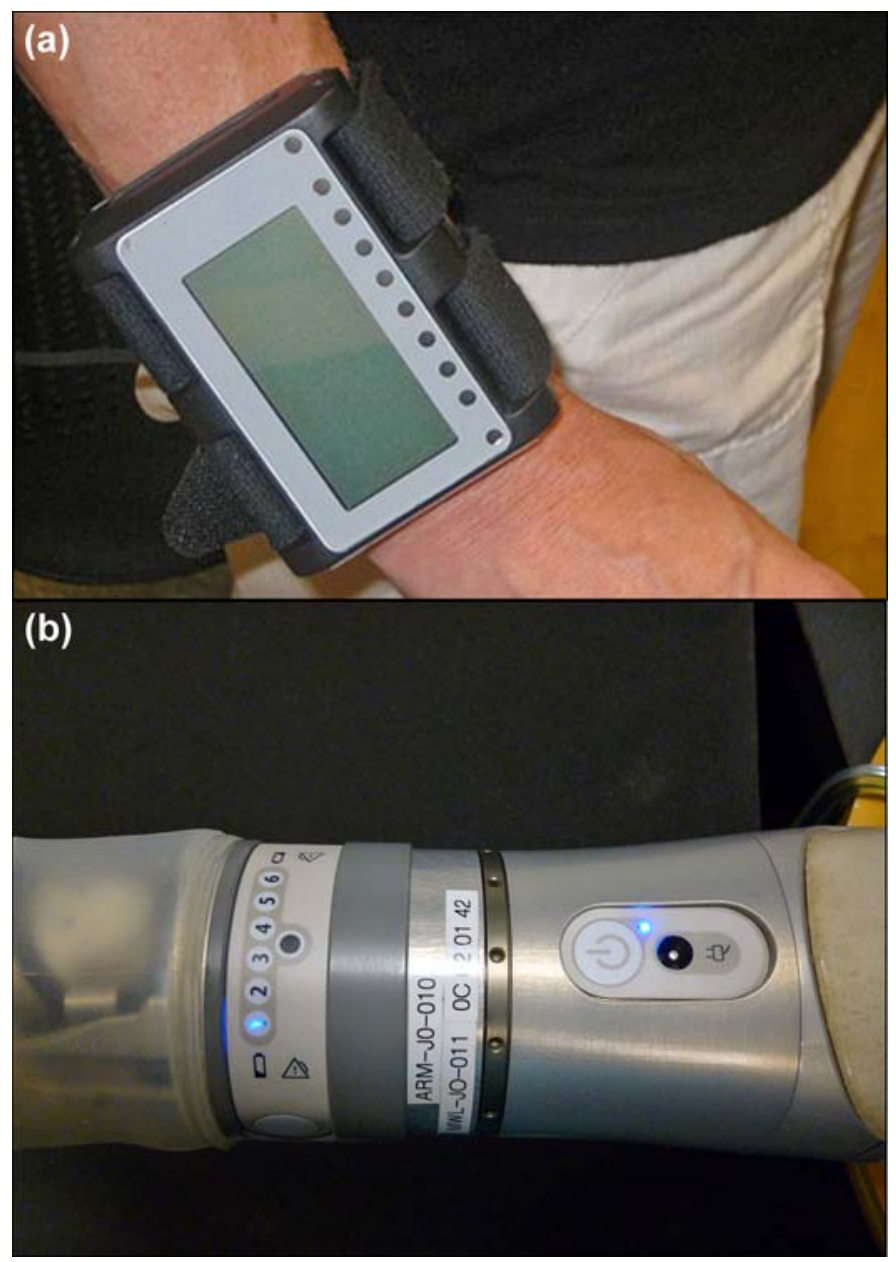

Figure 3.

Visual notification system. (a) Luke User Interface worn on wrist (second-generation DEKA Arm). (b) Wrist display embedded into third-generation DEKA Arm wrist. rizes the major subject and clinician recommendations to improve the gen 2 device and reports on subjects' satisfaction and usability ratings for the gen 2 and gen 3 prototypes. Detailed qualitative feedback on subject perceptions about the DEKA Arm and its controls are reported in separate articles.

\section{METHODS}

Five sites participated in this study between 2009 and 2012: Providence VA Medical Center, Providence, Rhode Island; VA New York Harbor Healthcare System (NYHHS), Brooklyn, New York; James A. Haley Veterans' Hospital, Tampa, Florida; VA Long Beach Healthcare System, Long Beach, California; and the Center for the Intrepid (CFI), San Antonio, Texas. The protocol was approved by the institutional review boards at each site. Subjects met the following inclusion criteria: at least $18 \mathrm{yr}$ old and single or bilateral upper-limb amputation at the TR, TH, SD, or scapulathoracic level. All subjects were required to have active control over one or both ankles or have an appropriate number of myoelectric and/ or other control sites to enable adequate prosthetic control configuration for the DEKA Arm (as determined by the study prosthetist). Subjects were excluded if they had significant uncorrectable visual deficits; major communication or neurocognitive deficits; skin conditions that prevented prosthetic wear; an electrically controlled medical device; or any significant comorbidity, cognitive deficit, or mental health problem that would limit their ability to participate fully.

Subjects were recruited through clinical staff, flyers and brochures, email lists, and press releases. All subjects provided their written consent before entering the study.

After DEKA Arm controls were set up, subjects were familiarized with controls and arm features by using the virtual reality environment, an interactive computer software program [13]. Subjects were then trained in use of the device over the course of 10 or 15 two hour training sessions, depending on level of amputation. Prosthetic use training began with reinforcement of prosthetic control patterns of motions, proceeded to simple grasp and release activities, and progressed to more complex functional tasks. The training protocol for SC subjects was extended from 10 visits to 15 visits partway through the study, after a need for additional training for users at this level was recognized. 


\section{Data Collection}

\section{Recommendations for Improvements}

Subject and clinician recommendations for improvements to the DEKA Arm were gathered using several methods. First, all study sessions were videotaped, and subject and clinician feedback was made during study sessions, videos were viewed by study analysts, and key recommendations were extracted from the sessions. Subjects and clinicians also had use of digital audio recorders that they could use after study sessions to make comments about their experiences and the DEKA Arm and suggestions for how it could be optimized. Audiotapes were transcribed verbatim and analyzed for recommendations. In addition, structured surveys containing both structured and open-ended questions eliciting critical feedback and recommendations for improvement were administered after fitting the DEKA Arm, after $10 \mathrm{~h}$ of training, and at the end of training. At the end of the study, semistructured interviews were administered to elicit comments on areas not addressed in the end of study survey. Study prosthetists and therapists provided ongoing feedback and answered survey questions at the end of each subject's protocol.

\section{Standardized Tests and Measures}

The Trinity Amputation and Prosthesis Experience Scale (TAPES) satisfaction scale [16], the DEKA Arm satisfaction scale, and the DEKA Arm usability scale scores were collected at the end of the study. The TAPES was scored as overall composite and as three separate subscales: aesthetic satisfaction, weight satisfaction, and functional satisfaction [16]. Three DEKA Arm satisfaction scales and three DEKA Arm usability scales were developed to address the unique features of the DEKA Arm. Detailed information on the development and content of these measures is provided in the Appendix (available online only).

Briefly, the gen 2-gen 3 satisfaction scale is a 25-item scale, with an alpha of 0.89 , that was administered to both gen 2 and gen 3 subjects. Two additional items, also administered to both gen 2 and gen 3 subjects, that did not fit well with the overall scale were examined individually. A gen 2 satisfaction scale, with an alpha of 0.63 , was constructed from three items that were asked only in the gen 2 portion of the study. A 15-item gen 3 satisfaction scale, with an alpha of 0.87 , was used in the gen 3 portion of the study. Five additional items that did not fit well with the gen 3 satisfaction score were evaluated individually. In all satisfaction scales developed for this study, subjects were asked to rate satisfaction with specific aspects of the DEKA Arm's function on a 7-point scale (where 1 = very unhappy, 2 = unhappy, 3 = mostly dissatisfied, $4=$ mixed, $5=$ mostly satisfied, $6=$ happy, and $7=$ very happy).

The 17-item gen 2-gen 3 usability scale, with alpha of 0.89 , was administered to subjects in the gen 2 and gen 3 portions of the study. Six items that did not fit well with the overall scale were not included in the summary score and were examined individually. The gen 2 usability scale included three items, with an alpha of 0.54 , that were asked to gen 2 users only. Two items that did not fit well with the overall scale were examined individually. The gen 3 usability scale, with alpha of 0.85 , contained five items that were asked only to gen 3 users. Four additional items that did not fit well with the overall scale were examined individually. All usability scales used a 6-point scale (where 1 = unable to do, 2 = very difficult, 3 = difficult, 4 = neither easy nor difficult, 5 = easy, and $6=$ very easy).

\section{Data Analysis}

At the conclusion of each subject's participation in the study, all recommendations for optimizing the DEKA Arm were identified from data sources including video, audio, surveys, and semiguided interviews; they were then grouped by theme. A written synopsis of usability concerns and recommendations was then sent to the study site for verification by clinicians. After verification, and refinement as needed, the usability report with recommended improvements was forwarded to DEKA. At the conclusion of the study, themes from all usability reports were compiled by investigators and the VA research team evaluated whether or not study recommendations identified in gen 2 usability reports had been addressed in whole or in part in the gen 3 design.

Users' perspectives on usability and satisfaction with the gen 2 and gen 3 prototypes were evaluated using the TAPES and the usability and satisfaction scales and items developed for this study. Descriptive statistics for all scales and individual items were examined and results compared by prototype using nonparametric Wilcoxon rank-sum tests. Results for summary scores were stratified by level of DEKA Arm and outcomes compared by prototype using Kruskal-Wallis analysis of ranks tests. 


\section{RESULTS}

Thirty-nine subjects participated in the study: 26 in the gen 2 phase and 13 in the gen 3 phase. Of the 13 gen 3 subjects, 5 had participated in the gen 2 phase. Two subjects from gen 2 did not complete the end of study survey questions because they terminated the study unex- pectedly. Table 1 shows detailed characteristics of the 37 subjects who provided end of study survey data and are included in this analysis. Of these gen 2 subjects, 8 were fit with an RC, 6 with an HC, and 10 with an SC. Of the gen 3 subjects, 4 were fit with an RC, 5 with an HC, and 4 with an SC. There were four female subjects in the gen 2 group and one in the gen 3 group.

Table 1.

Characteristics of subjects included in analysis of second-generation (gen 2) and third-generation (gen 3) DEKA Arms.

\begin{tabular}{|c|c|c|}
\hline Characteristic & Gen $2(n=24)$ & Gen $3(n=13)$ \\
\hline \multicolumn{3}{|l|}{$\overline{\text { Age (yr) }}$} \\
\hline Mean \pm SD & $44.8 \pm 17.0$ & $46.4 \pm 16.4$ \\
\hline Range & $19.7-82.8$ & $23.1-70.8$ \\
\hline \multicolumn{3}{|l|}{ Training Visits $(n)$} \\
\hline Mean \pm SD & $10.3 \pm 3.0$ & $10.7 \pm 3.5$ \\
\hline Range & $5-15$ & $5-15$ \\
\hline \multicolumn{3}{|l|}{ DEKA Arm Fit Level, $n$ (\%) } \\
\hline Radial Configuration & $8(33.3)$ & $4(30.8)$ \\
\hline Humeral Configuration & $6(25.0)$ & $5(38.5)$ \\
\hline Shoulder Configuration & $10(41.7)$ & $4(30.8)$ \\
\hline \multicolumn{3}{|l|}{ Sex, $n(\%)$} \\
\hline Male & $20(83.3)$ & $12(92.3)$ \\
\hline Female & $4(16.7)$ & $1(7.7)$ \\
\hline \multicolumn{3}{|l|}{ Race, $n(\%)$} \\
\hline White & $21(87.5)$ & $13(100.0)$ \\
\hline Other & $3(12.5)$ & $0(0.0)$ \\
\hline \multicolumn{3}{|l|}{ Veteran, $n(\%)$} \\
\hline Nonveteran & $7(29.7)$ & $5(38.5)$ \\
\hline Veteran & $12(50.0)$ & $5(38.5)$ \\
\hline Active Duty & $5(20.8)$ & $3(23.1)$ \\
\hline \multicolumn{3}{|c|}{ Prosthetic User (active device only), $n$ (\%) } \\
\hline Not Current User & $2(8.3)$ & $2(15.4)$ \\
\hline Full-Time & $13(54.2)$ & $6(46.2)$ \\
\hline Part-Time & $9(37.5)$ & 5 (38.5) \\
\hline Participant in Gen 2 Study, $n$ (\%) & $24(100.0)$ & $5(38.5)$ \\
\hline \multicolumn{3}{|c|}{ Prosthetic Experience (includes cosmetic), $n$ (\%) } \\
\hline Not Prosthetic User & $2(8.3)$ & $2(15.4)$ \\
\hline Very New $(<3$ mo $)$ & $1(4.5)$ & $0(0.0)$ \\
\hline New (3 mo-1 yr) & $4(18.2)$ & $3(27.3)$ \\
\hline Experienced (1-5 yr) & $3(13.6)$ & $5(45.5)$ \\
\hline Very Experienced ( $\geq 5$ yr) & $14(63.6)$ & $3(27.3)$ \\
\hline
\end{tabular}


Summary of Gen 2 and Gen 3 Recommendations for Improvement

Table 2 summarizes the major user and clinician suggestions for improvements to gen 2 and indicates whether or not the research team believed that these suggestions were addressed in the gen 3 design. With few exceptions, the areas of improvement recommended by gen 2 users and clinicians were addressed to some degree in gen 3 . Clinicians also provided detailed feedback about the computer software used to configure the controls. This feedback was shared with DEKA on an ongoing basis but is not detailed in Table 2 or in this article.

\section{Overall Satisfaction and Perceived Usability}

Quantitative analyses suggest that satisfaction and perceived usability of the DEKA Arm was greater for gen 3 than for gen 2. Overall TAPES scores were similar for gen 2 and gen 3 (Table 3); however, scores of the TAPES aesthetic satisfaction subscale were higher for

Table 2.

Summary of recommended improvements (second-generation DEKA Arm [gen 2]) and changes to third-generation DEKA Arm (gen 3).

\begin{tabular}{|c|c|c|c|}
\hline \multirow{2}{*}{ Recommendation } & \multicolumn{2}{|c|}{ Gen 2} & \multirow{2}{*}{ Addressed in Gen 3? } \\
\hline & User & Clinician & \\
\hline \multicolumn{4}{|l|}{ Size/Weight } \\
\hline Make lighter in weight. & $x$ & $x$ & No \\
\hline Make arm/hand smaller/less bulky. & $x$ & $x$ & Yes \\
\hline \multicolumn{4}{|l|}{ Comfort/Public Use } \\
\hline \multicolumn{4}{|l|}{ Reduce components/wires (number and size). } \\
\hline Reduce external components. & $x$ & $\times$ & Yes \\
\hline Reduce wires. & $x$ & $x$ & Yes \\
\hline Internalize battery. & & $x$ & Yes $^{*}$ \\
\hline Make wireless or internalize wires. & $x$ & $x$ & Yes \\
\hline Improve cosmesis. & $x$ & & Yes \\
\hline Make less noisy. & $x$ & & Yes \\
\hline Waterproof. & $x$ & & Yes \\
\hline Improve inflatable socket bladders. & $x$ & & Yes \\
\hline \multicolumn{4}{|l|}{ Controls/User Interface } \\
\hline Improve foot controls. & $x$ & $x$ & Yes \\
\hline Improve EMG system. & $x$ & $\times$ & Yes \\
\hline Improve user notification system. & $\times$ & $x$ & Yes \\
\hline Make process of correcting faults easier. & $x$ & $\times$ & Yes \\
\hline Improve tactor. & $x$ & $x$ & No \\
\hline Improve battery life (main/IMU). & & $x$ & Yes \\
\hline \multicolumn{4}{|l|}{ Mechanics/Movements } \\
\hline Wrist: Add radial-ulnar deviation/increase tension. & $x$ & $x$ & Yes \\
\hline Improve grip force/finger alignment/grip speed. & $x$ & & Yes \\
\hline Improve end-point control. & & $x$ & Yes \\
\hline \multicolumn{4}{|l|}{ Reliability } \\
\hline Improve overall reliability. & $x$ & $x$ & Yes \\
\hline Improve reliability of hand and fingers. & $x$ & $x$ & Yes \\
\hline Reduce faults and unexplained stoppages. & $x$ & $x$ & Yes \\
\hline
\end{tabular}

${ }^{*}$ For humeral and shoulder configurations.

EMG = myoelectrode, IMU = inertial measurement unit. 
Table 3.

Satisfaction and usability ratings (mean \pm standard deviation) by second-generation (gen 2) and third-generation (gen 3) DEKA Arm prototype at end of study.

\begin{tabular}{|c|c|c|c|}
\hline Measure & Gen $2(n=21)$ & Gen $3(n=11)$ & $p$-Value \\
\hline TAPES & $3.3 \pm 0.8$ & $3.6 \pm 0.4$ & 0.45 \\
\hline Aesthetic & $3.2 \pm 0.8$ & $3.9 \pm 0.6$ & $0.04^{*}$ \\
\hline Weight & $2.4 \pm 1.2$ & $2.4 \pm 1.4$ & 0.71 \\
\hline Function & $3.7 \pm 0.9$ & $3.6 \pm 0.6$ & 0.70 \\
\hline Scale & Gen $2(n=24)$ & Gen $3(n=13)$ & $p$-Value \\
\hline Gen 2-Gen 3 Satisfaction & $5.3 \pm 0.8$ & $5.6 \pm 0.7$ & 0.18 \\
\hline
\end{tabular}

gen $3(p=0.04)$, indicating greater satisfaction with the appearance of the device. No statistically significant differences were observed in TAPES scores for overall scale or subscales when stratified by DEKA Arm level (results not shown).

Scores of the gen 2-gen 3 satisfaction scale were higher for gen 3, but differences were not statistically significant. No statistically significant differences were observed in DEKA Arm satisfaction scores when stratified by DEKA Arm level (results not shown). Scores of individual satisfaction items that were significantly higher for gen 3 subjects than for gen 2 subjects related to satisfaction with device doffing, grip switching, elbow movement, and humeral rotation (Table 4). Among all items asked of gen 3 subjects, those with the lowest mean satisfaction scores ( $3=$ mostly dissatisfied) were satisfaction with wires and cables, rated as 3.3, followed by weight of the arm, rated as 3.5. The three highest rated items (6 = happy) were battery charger, rated at 6.5; shoulder appearance, rated at 6.3; and EMG speed, rated at 6.2 .

Scores for the gen 2-gen 3 usability scale were higher for gen 3 , indicating better overall perceived usability for the gen 3 prototype $(p=0.02)$. No statistically significant differences were observed in these scores when stratified by DEKA Arm level (results not shown). Scores of individual usability items showed higher rankings for the following items in Gen 3: socket and harness doffing, chuck grip, tool grip, pinch grip, lateral pinch, power grip, and grip switching $(p<0.05)$ (Table 5).

The mean score of the 3 -item satisfaction scale asked only in gen 2 was 5.4 and the mean score for the 3-item gen 2 usability scale was 5.3 (Table 6). The mean score of the gen 3 satisfaction scale was 4.8 and the mean score for the gen 3 usability scale was 4.7. The lowest ranked usability items asked only to gen 3 subjects were related to fingernails, rated as 4.2 , followed by hand covering, rated as 4.3, and the DEKA Arm system as a whole, rated as 4.3 (Table 6). The highest ratings were for the battery charger, rated at 5.7; tactor for mode, rated at 5.8; and tactor for grip, rated at 5.8.

\section{DISCUSSION}

This article summarized subjects' and clinicians' key recommendations to improve the gen 2 DEKA Arm, and presented subjects' ratings of satisfaction and usability. Subjects' ratings of satisfaction and usability were compared by DEKA Arm prototype using standardized measures. Our findings indicate that subjects rated aspects of satisfaction and usability higher for the gen 3 than for the gen 2 prototype. Data suggests that DEKA's optimization efforts were successful, although there are features of the gen 3 , such as its weight, external cables and wires, reliability, hand covering, and fingernails, that would benefit from further optimization efforts.

Satisfaction with the gen 3 appearance, as measured by the TAPES, was rated higher than that of the gen 2 . Considerable efforts were made to enhance the appearance of the gen 3 prototype by altering the contours and arm covering, miniaturizing the IMUs, eliminating the ankle straps, and embedding the wrist display.

Users of both prototypes rated their satisfaction with the DEKA Arm as "mostly satisfied" on the gen 2-gen 3 satisfaction scale developed for this study. Comparison of individual items from the scale revealed that gen 3 users were more satisfied with doffing, grip switching, and 
Table 4.

Comparison (mean \pm standard deviation) of satisfaction items asked of second-generation (gen 2) and third-generation (gen 3) DEKA Arm users.

\begin{tabular}{|c|c|c|c|}
\hline Item & Gen $2(n=24)$ & Gen $3(n=13)$ & $p$-Value \\
\hline Overall Function & $5.1 \pm 1.6$ & $5.2 \pm 1.6$ & 0.91 \\
\hline Inertial Measurement Units & $4.8 \pm 1.1$ & $5.2 \pm 1.7$ & 0.40 \\
\hline Other Controls & $5.2 \pm 1.6$ & $5.8 \pm 1.3$ & 0.61 \\
\hline Tactor for Grip Pressure & $4.6 \pm 1.7$ & $4.9 \pm 1.7$ & 0.57 \\
\hline Virtual Reality Environment & $5.4 \pm 1.2$ & $5.0 \pm 1.5$ & 0.47 \\
\hline Donning & $4.3 \pm 2.0$ & $5.2 \pm 1.5$ & 0.20 \\
\hline Doffing & $4.5 \pm 2.0$ & $5.8 \pm 1.3$ & $0.03^{*}$ \\
\hline Hand Operation & $5.9 \pm 1.4$ & $5.9 \pm 1.0$ & 0.79 \\
\hline Chuck Grip & $5.3 \pm 1.5$ & $5.2 \pm 1.4$ & 0.69 \\
\hline Tool Grip & $5.3 \pm 1.3$ & $5.8 \pm 1.1$ & 0.20 \\
\hline Pinch Grip & $5.6 \pm 1.1$ & $6.1 \pm 1.4$ & 0.08 \\
\hline Lateral Pinch & $5.9 \pm 1.1$ & $5.5 \pm 1.1$ & 0.25 \\
\hline Power Grip & $5.7 \pm 1.3$ & $6.3 \pm 0.8$ & 0.20 \\
\hline Switching Grips & $4.9 \pm 1.7$ & $5.9 \pm 1.8$ & $0.03^{*}$ \\
\hline Forearm Rotation & $5.5 \pm 1.5$ & $6.3 \pm 1.0$ & 0.10 \\
\hline Wrist Movement & $5.2 \pm 1.5$ & $5.8 \pm 1.3$ & 0.22 \\
\hline Elbow Movement & $5.3 \pm 1.2$ & $6.3 \pm 0.9$ & $0.05^{*}$ \\
\hline Humeral Rotation & $5.3 \pm 1.1$ & $6.3 \pm 1.1$ & $0.04^{*}$ \\
\hline Socket Comfort & $5.3 \pm 1.7$ & $5.5 \pm 1.1$ & 0.15 \\
\hline Harnessing & $5.0 \pm 1.8$ & $4.8 \pm 1.2$ & 0.57 \\
\hline Bladders & $5.1 \pm 1.5$ & $5.6 \pm 1.3$ & 0.39 \\
\hline Socket Stability & $5.5 \pm 1.4$ & $6.0 \pm 0.9$ & 0.45 \\
\hline Grip Indicator & $5.6 \pm 1.2$ & $5.3 \pm 1.5$ & 0.69 \\
\hline Mode Indicator & $5.6 \pm 1.4$ & $5.9 \pm 1.2$ & 0.63 \\
\hline Battery Indicator & $5.3 \pm 1.4$ & $6.2 \pm 1.1$ & 0.11 \\
\hline \multicolumn{4}{|c|}{ Not Included in Summary Score } \\
\hline Myoelctrodes & $4.9 \pm 1.5$ & $5.4 \pm 1.0$ & 0.32 \\
\hline Error Indicator & & $6.0 \pm 1.3$ & 0.41 \\
\hline
\end{tabular}

movements of the elbow and upper arm than gen 2 users. Greater satisfaction with switching grips may have been associated with the gen 3 wrist display, which provides user notification of current handgrip.

Overall usability ratings were higher for gen 3 users who indicated that using the arm was "easy" as compared with gen 2 users who indicated that it was "neither easy nor difficult." All of the grips were rated as more usable by gen 3 users than gen 2 users. This may be because the grip trajectory and finger shapes were changed in gen 3 . Gen 3 users also rated the elbow and humeral movements as more usable than did gen 2 users.

The findings reported in this article can be triangulated with the findings from the qualitative analysis that will be reported elsewhere, which provides further insights into why users may have rated satisfaction and usability the way that they did.

To our knowledge, ours is largest evaluation study of any new upper-limb prosthetic technology ever conducted. We worked with the device developer (DEKA) throughout the gen 3 development phase to provide feedback on specific device features. We then evaluated subject feedback on the resulting gen 3 design. Our findings can be used to further refine the gen 3 prototype and prepare it for commercialization. The integration of findings from usability research into the product development process should result in a better final product. Moreover, our findings can be used by other device developers who want to understand user perspectives on specific device features and functions. 
JRRD, Volume 51, Number 1, 2014

Table 5.

Comparison (mean \pm standard deviation) of usability items asked of second-generation (gen 2) and third-generation (gen 3) DEKA Arm users.

\begin{tabular}{|c|c|c|c|}
\hline Item & Gen $2(n=24)$ & Gen $3(n=13)$ & $p$-Value \\
\hline Overall Function & $4.8 \pm 1.0$ & $5.1 \pm 1.0$ & 0.37 \\
\hline Inertial Measurement Units & $4.6 \pm 0.9$ & $5.2 \pm 1.0$ & 0.13 \\
\hline Tactor for Grip Pressure & $4.8 \pm 1.2$ & $5.3 \pm 1.0$ & 0.21 \\
\hline Donning & $3.9 \pm 1.7$ & $4.5 \pm 1.7$ & 0.15 \\
\hline Doffing & $4.0 \pm 1.5$ & $5.0 \pm 1.8$ & $0.02^{*}$ \\
\hline Tool Grip & $5.0 \pm 0.7$ & $5.6 \pm 0.5$ & $0.02^{*}$ \\
\hline Pinch Grip & $5.1 \pm 0.7$ & $5.8 \pm 0.4$ & $<0.01^{*}$ \\
\hline Lateral Pinch & $5.1 \pm 0.8$ & $5.7 \pm 0.5$ & $0.01^{*}$ \\
\hline Power Grip & $5.1 \pm 0.8$ & $5.7 \pm 0.5$ & $0.02^{*}$ \\
\hline Switching Grips & $4.6 \pm 1.1$ & $5.4 \pm 1.0$ & $0.02^{*}$ \\
\hline Humeral Rotation & $4.9 \pm 0.7$ & $5.6 \pm 0.5$ & $0.05^{*}$ \\
\hline Harnessing & $4.1 \pm 1.7$ & $4.3 \pm 1.9$ & 0.68 \\
\hline \multicolumn{4}{|l|}{ Not Included in Summary Score } \\
\hline Myoelectrodes & $5.0 \pm 1.0$ & $4.8 \pm 1.1$ & 0.58 \\
\hline Virtual Reality Environment & & $5.1 \pm 0.8$ & 0.34 \\
\hline Bladders & $4.8 \pm 1.3$ & $4.9 \pm 1.8$ & 0.68 \\
\hline Grip Indicator & & $5.5 \pm 0.7$ & 0.74 \\
\hline Mode Indicator & $5.5 \pm 1.0$ & $5.4 \pm 0.7$ & 0.51 \\
\hline Battery Indicator & & $5.5 \pm 0.7$ & 0.85 \\
\hline
\end{tabular}

Although we compared mean scores for some scales and some items by prototype, we recognize that our results need to be interpreted cautiously for several reasons. We tested two groups of subjects: those who participated in gen 2 and those who participated in gen 3 . These two groups may have differed from each other in ways that could have influenced the satisfaction and usability ratings. The few individuals who participated in both the gen 2 and gen 3 portions of the study were in the best position to evaluate improvements in the prototypes. Therefore, we examined summary scores for the five subjects who participated in both the gen 2 and gen 3 portions of the study and found that their ratings of satisfaction and usability were higher in gen 3 for all scales, although only statistically different for the TAPES aesthetic satisfaction scale.

Furthermore, our analyses are limited by the small sample size. Because we lacked the statistical power to detect differences in groups, we were at risk of making a type II statistical effort, meaning that we may have failed to detect a true difference in scores when one did indeed exist. This might explain why several of the key improvements made in the gen 3 (such as changes to the IMUs and indicators) were rated more highly, but differences in scores were not statistically significant. We conducted a post hoc power analysis and found that we would have needed two equal size samples of 23 persons in each to be 80 percent confident that we could detect a large effect size. Such a sample size was not feasible given the limitations of funding and the time line of DEKA's optimization efforts.

\section{CONCLUSIONS}

Data suggest that DEKA's optimization efforts were successful. Compared with gen 2 users, gen 3 users were more satisfied with appearance, grips, elbow movement, 
Table 6.

Items and scales utilized in second-generation (gen 2) or thirdgeneration (gen 3) DEKA Arms only.

\begin{tabular}{|c|c|c|}
\hline Satisfaction & $n^{*}$ & Mean \pm SD \\
\hline \multicolumn{3}{|l|}{ Gen 2 Only } \\
\hline Force Sensitive Resistors & 6 & $4.5 \pm 1.6$ \\
\hline Air Bladder Controls & 19 & $5.8 \pm 1.1$ \\
\hline Dynamic Straps & 6 & $5.0 \pm 1.8$ \\
\hline Summary Score & 21 & $5.4 \pm 1.4$ \\
\hline \multicolumn{3}{|l|}{ Gen 3 Only } \\
\hline Arm Appearance & 13 & $5.5 \pm 1.0$ \\
\hline Hand Shape & 13 & $5.5 \pm 1.1$ \\
\hline Hand Size & 13 & $5.1 \pm 1.7$ \\
\hline Hand Covering & 12 & $5.2 \pm 1.2$ \\
\hline Arm System & 13 & $3.8 \pm 1.9$ \\
\hline Hardware Reliability & 13 & $4.6 \pm 1.4$ \\
\hline Hand Cover Durability & 13 & $4.8 \pm 1.3$ \\
\hline Hand Cover Material & 13 & $4.6 \pm 1.1$ \\
\hline Fingernails & 13 & $4.5 \pm 1.6$ \\
\hline Inertial Measurement Unit Speed & 13 & $5.7 \pm 1.3$ \\
\hline Tactor for Mode & 9 & $5.7 \pm 0.7$ \\
\hline Tactor for Grip Change & 10 & $5.7 \pm 0.7$ \\
\hline Weight & 13 & $3.5 \pm 1.7$ \\
\hline Wires and Cables & 13 & $3.3 \pm 1.8$ \\
\hline Waterproofing & 8 & $5.4 \pm 1.3$ \\
\hline Summary Score & 13 & $4.8 \pm 0.8$ \\
\hline \multicolumn{3}{|c|}{ Items Not Used in Gen 3 Summary Score } \\
\hline Shoulder Appearance & 4 & $6.3 \pm 1.0$ \\
\hline Myoelectrode Speed & 9 & $6.2 \pm 0.4$ \\
\hline End-Point Control & 4 & $6.0 \pm 0.8$ \\
\hline Dynamic Socket Controller & 6 & $5.7 \pm 1.0$ \\
\hline Battery Charger & 11 & $6.5 \pm 0.5$ \\
\hline Usability & $n$ & Mean \pm SD \\
\hline \multicolumn{3}{|l|}{ Gen 2 Only } \\
\hline Hand Operation Use & 24 & $5.4 \pm 0.9$ \\
\hline Air Bladder Controls & 19 & $5.4 \pm 0.6$ \\
\hline Dynamic Straps & 6 & $4.7 \pm 1.2$ \\
\hline Summary Score & 24 & $5.3 \pm 0.9$ \\
\hline \multicolumn{3}{|c|}{ Items Not Used in Gen 2 Summary Score } \\
\hline Force Sensitive Resistors & 6 & $4.3 \pm 1.2$ \\
\hline Socket Stability & 23 & $4.9 \pm 1.2$ \\
\hline \multicolumn{3}{|l|}{ Gen 3 Only } \\
\hline Hand Covering & 9 & $4.3 \pm 0.9$ \\
\hline Hand Cover Material & 12 & $4.5 \pm 1.1$ \\
\hline Fingernails & 13 & $4.2 \pm 1.3$ \\
\hline Dynamic Socket Controller & 6 & $5.3 \pm 0.8$ \\
\hline Battery Charger & 11 & $5.7 \pm 0.5$ \\
\hline Summary Score & 13 & $4.7 \pm 0.7$ \\
\hline \multicolumn{3}{|c|}{ Items Not Used in Gen 3 Summary Score } \\
\hline Arm System & 12 & $4.3 \pm 0.9$ \\
\hline Tactor for Mode & 9 & $5.8 \pm 0.4$ \\
\hline Tactor for Grip Change & 10 & $5.8 \pm 0.4$ \\
\hline End-Point Control & 4 & $5.3 \pm 0.5$ \\
\hline
\end{tabular}

${ }^{*}$ Number of respondents who completed item. Not all items were applicable to all subjects.

$\mathrm{SD}=$ standard deviation . and doffing and rated overall usability higher. There are still features of the gen 3, including weight and external cables and wires, that would benefit from further optimization efforts.

\section{ACKNOWLEDGMENTS}

\author{
Author Contributions: \\ Study concept and design: L. Resnik. \\ Analysis and interpretation of data: L. Resnik, M. Borgia. \\ Drafting of manuscript: L. Resnik, M. Borgia. \\ Critical revision of manuscript for important intellectual content: \\ L. Resnik, M. Borgia. \\ Statistical analysis: L. Resnik, M. Borgia. \\ Obtained funding: L. Resnik. \\ Study supervision: L. Resnik.
}

Financial Disclosures: The authors have declared that no competing interests exist.

Funding/Support: This material was based on work supported by VA RR\&D (grants A6780 and A6780I). DEKA's support of the VA Optimization Study was sponsored by DARPA and the U.S. Army Research Office.

Additional Contributions: As of the publication date of this article, the DEKA Arm system is currently being reviewed by the U.S. Food and Drug Administration and has not received clearance to be commercially marketed. The authors acknowledge the valuable work of study coordinator Kate Barnabe and members of the research teams at all study sites: VA NYHHS (Nicole Sasson, MD; Christopher Fantini, CP, MSPT; Kenneth Breuer, CP; Roxanne Disla, OTR/L; Mary Anne Garbarini, MA, PT; Dan Burgos, BOCP; and Edward Sliwinski), James A. Haley Veterans' Hospital (Gail Latlief, DO; Melanie Harris, CPO; Samuel Phillips, PhD, CP, FAAOP; Laurel Adams-Koss, MOT, OTR/L; Deborah Gavin-Dreschnack, PhD; Jemy Delikat, MOT, OTR; Jill Ardilla, MA; Andrea Spehar, DVM, MPH, JD; N. Joseph Shamp, CPO; and Steve Doerr, CPO), CFI (Lisa Smurr-Walters MS, OTR/L, CHT; Ryan Blanck, LCPO; Kathryn Korp, OTD, OTR/L; Sandra Jarzombek, MA; John Fergason, CPO; Christopher Ebner, MS, OTR/L; COL Jennifer Menetrez, MD; and Donald A. Gajewski, MD), VA Long Beach Healthcare System (Dana Craig; Susan Kaplan, MD; Karen Duddy, MHA, OTR/L; Jack Mark, CPO; Dorene Doi, OTR/L; Mary Jo Van Duyn; and Duane Sallade, CPO), and Providence VA Medical Center (Susan Rizzo, MPH; Marcia Selinger; Crystal Davis, MPH; Debra Kelty, MPA; Shana Klinger, MA; Katherine Etter, MS; Marissa Meucci, MS).

Institutional Review: The protocol was approved by the institutional review boards at each site. All subjects provided their written consent before entering the study.

Participant Follow-Up: The authors have no plans to inform participants of the publication of this study.

Disclaimer: The information in this article does not necessary reflect the position or policy of the U.S. Government; no official endorsement should be inferred. 


\section{REFERENCES}

1. Department of Veterans Affairs Office of Inspector General. Healthcare inspection: Prosthetic limb care in VA facilities. Report No. 11-02138-116. Washington (DC): VA Office of Inspector General; 2012.

2. Biddiss EA, Chau TT. Upper limb prosthesis use and abandonment: A survey of the last 25 years. Prosthet Orthot Int. 2007;31(3):236-57. [PMID:17979010] http://dx.doi.org/10.1080/03093640600994581

3. Wright TW, Hagen AD, Wood MB. Prosthetic usage in major upper extremity amputations. J Hand Surg Am. 1995; 20(4):619-22. [PMID:7594289] http://dx.doi.org/10.1016/S0363-5023(05)80278-3

4. Atkins DJ, Heard DC, Donovan WH. Epidemiologic overview of individuals with upper-limb loss and their reported research priorities. J Prosthet Orthot. 1996;8(1):2-11.

5. Dakpa R, Heger, H. Prosthetic management and training of adult upper limb amputees. Curr Orthopaed. 1997;11(3): 193-202.

6. Lake C, Dodson R. Progressive upper limb prosthetics. Phys Med Rehabil Clin N Am. 2006;17(1):49-72.

[PMID:16517345]

http://dx.doi.org/10.1016/j.pmr.2005.10.004

7. Herberts P, Körner L, Caine K, Wensby L. Rehabilitation of unilateral below-elbow amputees with myoelectric prostheses. Scand J Rehabil Med. 1980;12(3):123-28. [PMID:7209446]

8. MaGuire MT. Empowering prosthetics: A team approach to prosthetics and limb loss. Rehabil Manag. 2008;2008:4.

9. Bhaskaranand K, Bhat AK, Acharya KN. Prosthetic rehabilitation in traumatic upper limb amputees (an Indian perspective). Arch Orthop Trauma Surg. 2003;123(7):363-66. [PMID:12827395] http://dx.doi.org/10.1007/s00402-003-0546-4

10. Northwestern University Feinberg School of Medicine. Research in P\&O: Are we addressing clinically relevant problems? Report on the State-of-the-Science Meeting in Prosthetics and Orthotics. Chicago (IL): Northwestern University; 2006.

11. Defense Sciences Office. Revolutionizing prosthetics [Internet]. Arlington (VA): Defense Advanced Research
Projects Agency; 2013 [cited 2012 Dec 21]. Available from: http://www.darpa.mil/our work/dso/programs/ revolutionizing_prosthetics.aspx

12. Resnik L, Klinger SL, Etter K. The DEKA Arm: Its features, functionality, and evolution during the Veterans Affairs Study to optimize the DEKA Arm. Prosthet Orthot Int. 2013 Oct 22. Epub ahead of print. [PMID:24150930]

13. Resnik L, Etter K, Klinger SL, Kambe C. Using virtual reality environment to facilitate training with advanced upperlimb prosthesis. J Rehabil Res Dev. 2011;48(6):707-18. [PMID:21938657] http://dx.doi.org/10.1682/JRRD.2010.07.0127

14. Altobelli DE, Coulter S, Perry NC. Design considerations in upper extremity prostheses. 2011 Myoelectric Controls/ Powered Prosthetics Symposium; 2011 Aug 14-19; Fredericton, Canada.

15. Alley RD, Williams TW 3rd, Albuquerque MJ, Altobelli DE. Prosthetic sockets stabilized by alternating areas of tissue compression and release. J Rehabil Res Dev. 2011; 48(6):679-96. [PMID:21938655] http://dx.doi.org/10.1682/JRRD.2009.12.0197

16. Desmond DM, MacLachlan M. Factor structure of the Trinity Amputation and Prosthesis Experience Scales (TAPES) with individuals with acquired upper limb amputations. Am J Phys Med Rehabil. 2005;84(7):506-13. [PMID:15973087] http://dx.doi.org/10.1097/01.phm.0000166885.16180.63

Submitted for publication February 28, 2013. Accepted in revised form July 31, 2013.

This article and any supplementary material should be cited as follows:

Resnik L, Borgia M. User ratings of prosthetic usability and satisfaction in VA study to optimize DEKA Arm. J Rehabil Res Dev. 2014;51(1):15-26.

http://dx.doi.org/10.1682/JRRD.2013.02.0056

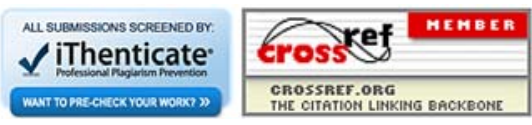

